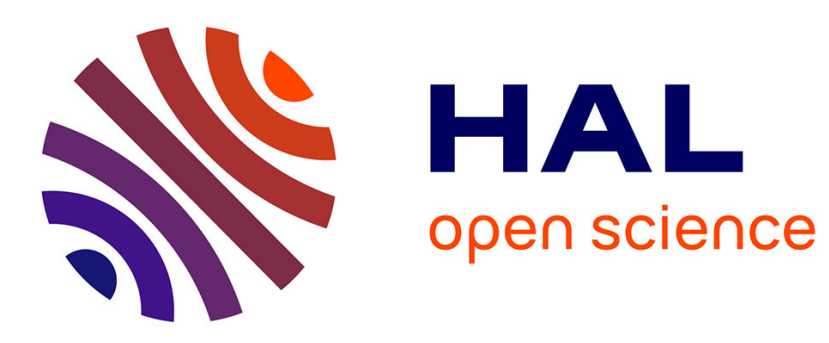

\title{
Time-domain response of damped stochastic multiple-degree-of-freedom systems
}

Eric Jacquelin, Denis Brizard, Sondipon Adhikari, Michael Ian Friswell

\section{To cite this version:}

Eric Jacquelin, Denis Brizard, Sondipon Adhikari, Michael Ian Friswell. Time-domain response of damped stochastic multiple-degree-of-freedom systems. Journal of Engineering Mechanics - ASCE, 2020, 146 (1), 21 p. 10.1061/(ASCE)EM.1943-7889.0001705 . hal-02459900

\section{HAL Id: hal-02459900 https://hal.science/hal-02459900}

Submitted on 29 Jan 2020

HAL is a multi-disciplinary open access archive for the deposit and dissemination of scientific research documents, whether they are published or not. The documents may come from teaching and research institutions in France or abroad, or from public or private research centers.
L'archive ouverte pluridisciplinaire HAL, est destinée au dépôt et à la diffusion de documents scientifiques de niveau recherche, publiés ou non, émanant des établissements d'enseignement et de recherche français ou étrangers, des laboratoires publics ou privés. 
JACQUELIN, Eric, BRIZARD, Denis, ADHIKARI, Sondipon, FRISWELL, Michael Ian, 2020, Time-domain response of damped stochastic multiple-degree-of-freedom systems, Journal of Engineering Mechanics, 146, 1, American Society of Civil Engineers ASCE, 21 p, DOI: 10.1061/(ASCE)EM.1943-7889.0001705

1 Time-domain response of damped stochastic multiple-degree-of-freedom systems

\section{ABSTRACT}

Characterizing the time-domain response of a random multiple-degree-of-freedom dynamical system is challenging and often requires Monte Carlo simulation (MCS). Differential equations must therefore be solved for each sample, which is time consuming. This is why polynomial chaos expansion (PCE) has been proposed as an alternative to MCS. However, it turns out that PCE is not adapted to simulate a random dynamical system for long-time integration. Recent studies have shown similar issues for the frequency response function of a random linear system around the deterministic eigenfrequencies. A Padé approximant approach has been successfully applied; similar interesting results were also observed with a random mode approach. Therefore the latter two methods were applied to a random linear dynamical system excited by a dynamic load to estimate the first two statistical moments and the probability density function at a given instant of time. Whereas the random modes method has been very efficient and accurate to evaluate the statistics of the response, the Padé approximant approach has given very poor results when the coefficients were determined in time domain. However, if the differential equations were solved in the frequency domain, the Padé 
approximants, which were also calculated in the frequency domain, provided results in excellent agreement with the MCS results.

KEYWORDS: Random dynamical systems; polynomial chaos expansion; transient response; random modes; extended Padé approximants.

\section{INTRODUCTION}

Over recent decades, numerical methods to solve nonlinear multiphysics multiscale models have been successfully developed. However the focus of these complex formulations has mainly concerned deterministic problems.

The challenge has been to account for the uncertainties to describe more accurately real systems. Neumann expansion (?), and polynomial chaos expansion (PCE) (Ghanem and Spanos 1991) can be considered as the first methods used to study random systems in an engineering context, and PC is now one of the most popular tools to propagate uncertainties through numerical models. Ghanem and Spanos (Ghanem and Spanos 1991) proposed to model the random parts of a quantity with a polynomial chaos (PC) expansion (PCE), which had been developed by Wiener (Wiener 1938). The original polynomial chaos has been extended to several families of random variables (?; Witteveen and Bijl 2006).

The objective of this work is to study random linear dynamical systems in the time domain. Polynomial chaos expansion has been already used to describe the time domain response of nonlinear random systems. It has been observed that accurate long-time integration would require a lot of terms in the expansion (Gerritsma et al. 2010; Le Maître et al. 2010) and is not effective in determining limit cycles of random oscillators (Beran et al. 2006; Le Meitour et al. 2010). Some methods have been proposed to improve the method, such as introducing a transformnation in the time domain (Le Maître et al. 2010; ?), identifying a random nonlinear autoregressive exogenous model (?), and updating the PC basis with a Gram-Schmidt orthogonalisation. This method aims to find a basis to describe the random response that leads to a low number of terms in the expansion.

A similar issue arises to evaluate the steady-state response around the mean natural frequencies of a random dynamical system. Methods based on Padé approximants (XPA) and random modes 
(RM) have been successfully applied to tackle this issue (Jacquelin et al. 2017). Therefore, it is interesting to explore the ability of these techniques to estimate the transient response of random linear dynamical systems.

First a brief presentation of the PCE, Padé approximants and random modes is given. The methods are then illustrated on an example. Finally conclusions are drawn.

\section{POLYNOMIAL CHAOS EXPANSION (PCE) IN THE TIME-DOMAIN}

A linear uncertain system is studied. The uncertainty is modelled with $r$ random variables, $\left\{\xi_{i}\right\}_{i=1, \cdots, r}$, gathered in vector $\boldsymbol{\Xi}$. The response, $\mathbf{x}(t, \boldsymbol{\Xi}) \in \mathbb{R}^{N}$, satisfies the following equation

$$
\mathbf{M}(\boldsymbol{\Xi}) \ddot{\mathbf{x}}(t, \boldsymbol{\Xi})+\mathbf{C}(\boldsymbol{\Xi}) \dot{\mathbf{x}}(t, \boldsymbol{\Xi})+\mathbf{K}(\boldsymbol{\Xi}) \mathbf{x}(t, \boldsymbol{\Xi})=\mathbf{F}(t)
$$

where $\mathbf{F}(t)$ is the force, and

$$
\mathbf{M}(\boldsymbol{\Xi})=\mathbf{M}_{0}+\sum_{i=1}^{r} \xi_{i} \mathbf{M}_{i}, \quad \mathbf{K}(\boldsymbol{\Xi})=\mathbf{K}_{0}+\sum_{i=1}^{r} \xi_{i} \mathbf{K}_{i}, \quad \mathbf{C}(\boldsymbol{\Xi})=\mathbf{C}_{0}+\sum_{i=1}^{r} \xi_{i} \mathbf{C}_{i}
$$

Random variables $\left\{\xi_{i}\right\}_{i=1 \cdots, r}$ are assumed to be independent, and to be zero mean. The system is deterministic when all the random variables are equal to zero, i.e. is defined with $\left(\mathbf{M}_{0}, \mathbf{K}_{0}\right.$, and $\left.\mathbf{C}_{0}\right)$.

In the following, only the stiffness matrix is assumed to be random, but the results can be easily obtained with random mass and damping matrices.

The solution of Eq. (1) is random and can be represented in terms of the PC basis $\left\{\Psi_{J}(\Xi), J \in \mathbb{N}\right\}$ (Ghanem and Spanos 1991) as

$$
\mathbf{x}(t, \Xi)=\sum_{J \in \mathbb{N}^{r}} \mathbf{Y}_{J}(t) \Psi_{J}(\Xi)
$$

where $J$ is a multi-index $J=\left(J_{1}, \cdots, J_{r}\right) ;|J|=\sum_{i=1}^{r} J_{i}$ is the order of polynomial $\Psi_{J}$. The PC basis are calculated from $r$ orthogonal polynomial sets $\left\{P_{J_{j}}\left(\xi_{j}\right)\right\}_{J_{j} \in \mathbb{N}}$ for $j=1, \cdots, r ; J_{j}$ is the order of $P_{J_{j}}\left(\xi_{j}\right)(?)$

$$
\Psi_{J}(\Xi)=\prod_{j=1}^{r} P_{J_{j}}\left(\xi_{j}\right)
$$


The choice of the $j$-th family $\left\{P_{J_{j}}\right\}$ is related to the probability distribution of $\xi_{j}$ (e.g., Legendre polynomial if $\xi_{j}$ has a uniform distribution): if the random variables follow a different statistical law, different families of $\mathrm{PC}$ are used. In the following the polynomials are normalized with respect to their probability distribution (see Eq. (8)).

For a numerical study, Eq. (3) is truncated at order $m$, with $P+1=(m+r) ! /(m ! r !)$ terms. Consequently the approximate solution $\mathrm{x}^{P}$ is an expansion over $\mathbb{I}_{m}$, a set of multi-index $J$ such that $\mathbb{I}_{m}=\left\{J \in \mathbb{N}^{r} /|J| \leq m\right\}$

$$
\mathbf{x}^{P}(t, \Xi)=\sum_{J \in \mathbb{I}_{m}} \mathbf{Y}_{J}(t) \Psi_{J}(\Xi)=\sum_{j=0}^{P} \mathbf{Y}_{j}(t) \Psi_{j}(\Xi) \Psi_{j}(\Xi)
$$

Exponent $P$ is no longer written explicitly to simplify the notation and the more tractable single index notation is used.

Substituting $\mathbf{x}$ from Eq. (5) into Eq. (1), and considering the orthogonality between the polynomials, Eq. (1) becomes

$$
\widetilde{\mathbf{M}} \ddot{\mathbf{Y}}(t)+\widetilde{\mathbf{C}} \dot{\mathbf{Y}}(t)+\widetilde{\mathbf{K}} \mathbf{Y}(t)=\widetilde{\mathbf{F}}(t)
$$

with 


$$
\mathbf{A}_{k} \in \mathbb{R}^{(P+1) \times(P+1)} \text { with }
$$

$$
\begin{aligned}
& {\left[\mathbf{A}_{0}\right]_{i j}=\int \ldots \int \Psi_{i}(\Xi) \Psi_{j}(\Xi) p(\Xi) \mathrm{d} \xi_{1} \ldots \mathrm{d} \xi_{r}=\delta_{i j}} \\
& {\left[\mathbf{A}_{k>0}\right]_{i j}=\int \cdots \int \xi_{k} \Psi_{i}(\Xi) \Psi_{j}(\Xi) p(\Xi) \mathrm{d} \xi_{1} \ldots \mathrm{d} \xi_{r}}
\end{aligned}
$$$$
\widetilde{\mathbf{M}}=\sum_{k=0}^{r} \mathbf{A}_{k} \otimes \mathbf{M}_{k} \in \mathbb{R}^{n(P+1) \times n(P+1)}
$$$$
\widetilde{\mathbf{C}}=\sum_{k=0}^{r} \mathbf{A}_{k} \otimes \mathbf{C}_{k} \in \mathbb{R}^{n(P+1) \times n(P+1)}
$$$$
\widetilde{\mathbf{K}}=\sum_{k=0}^{r} \mathbf{A}_{k} \otimes \mathbf{K}_{k} \in \mathbb{R}^{n(P+1) \times n(P+1)}
$$$$
\mathbf{Y}=\left[\begin{array}{lll}
\mathbf{Y}_{0}^{\top} & \mathbf{Y}_{1}^{\top} \ldots \mathbf{Y}_{P}^{\top}
\end{array}\right]^{\top} \in \mathbb{R}^{n(P+1)}
$$$$
\widetilde{\mathbf{F}}=\left[\begin{array}{llll}
\mathbf{F}^{\top} \mathbf{0} & \mathbf{0} & \ldots & \mathbf{0}
\end{array}\right]^{\top} \in \mathbb{R}^{n(P+1)}
$$

where $p(\Xi)$ is the joint probability density function $(\mathrm{PDF}), \delta_{i j}$ is the Kronecker delta, $\otimes$ is the

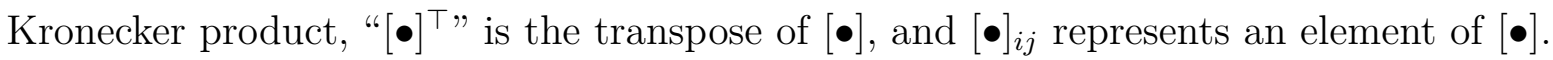

The frequency response function of such a system has been studied in several publications (?; ?; ?; ?; Jacquelin et al. 2015; ?; Yaghoubi et al. 2017). It was shown in (Jacquelin et al. 2015) that PCE provides excellent results except about the deterministic eigenfrequencies. Two methods based on PCE were proposed and proved their efficiency: the extended Padé approximants (XPA) and the random modes (RM).

As already mentioned, it has been highlighted in the literature that the PCE in the time domain loses its accuracy when the time increases, or many terms would be required in the expansion; this will be also verified in the following example. Therefore the Padé approximant approach and the random modes were applied to estimate the response and to test their efficiency in the time domain.

\section{PADÉ APPROXIMANTS AND RANDOM MODES}

\section{eXtended Padé Approximants (XPA): a rational function expansion}

Originally the Padé approximant (PA) technique consists in determining a rational function approximation from a known Taylor expansion of a function: it is supposed to converge much faster 
than the Taylor expansion (Baker and Graves-Morris 1996) when the function has poles.

The extended Padé approximants (XPA) are defined by replacing the monomials by PC in both the numerator and the denominator (Chantrasmi et al. 2009; Emmel et al. 2003; Guillaume et al. 2000; Matos 1996)

$$
\left[N_{k} / D_{k}\right]_{\mathbf{X}_{k}^{P C}}(t, \boldsymbol{\Xi})=\frac{\sum_{j=0}^{n_{k}} N_{k, j}^{X}{ }^{P A}(t) \Psi_{j}(\boldsymbol{\Xi})}{\sum_{j=0}^{d_{k}} D_{k, j}^{X P A}(t) \Psi_{j}(\boldsymbol{\Xi})}
$$

where $k$ refers to the $k$-th degree of freedom (DOF); $D_{k, 0}^{X P A}$ is equal to unity.

$N_{k, i}^{X P A}$ and $D_{k, i}^{X P A}$ are derived by comparing Eq. (5) to Eq. (15):

$$
\sum_{i=0}^{P} \mathbf{Y}_{i k}(t) \Psi_{j}(\boldsymbol{\Xi})=\frac{\sum_{j=0}^{n_{k}} N_{k, j}^{X P A}(t) \Psi_{j}(\boldsymbol{\Xi})}{1+\sum_{j=1}^{d_{k}} D_{k, j}^{X P A}(t) \Psi_{j}(\boldsymbol{\Xi})}
$$

Projecting Eq. (16) on a sufficient number of polynomial chaos gives the algebraic system verified by the unknown coefficients (Jacquelin et al. 2017).

\section{Random modes (RM)}

The response of a linear system can be determined with mode superposition as

$$
\mathbf{X}(t, \Xi)=\sum_{n=1}^{N} \widetilde{q}_{n}(t, \Xi) \widetilde{\boldsymbol{\phi}}_{n}(\Xi)
$$

where $\left\{\widetilde{\omega}_{k}, \widetilde{\phi}_{k}\right\}$ are the random modes, and $\widetilde{q}_{n}$ is the random modal coordinate.

The random modes can be estimate from the deterministic modes, $\left\{\omega_{k}, \phi_{k}\right\}$, and a PCE (Dessombz et al. 1999; Jacquelin et al. 2017):

$$
\begin{aligned}
& \widetilde{\omega}_{k}^{2}=\omega_{k}^{2}\left(\sum_{p=0}^{P} a_{p}^{k} \Psi_{p}(\boldsymbol{\Xi})\right) \\
& \widetilde{\boldsymbol{\phi}}_{k}=\sum_{n=1}^{N} \tilde{\lambda}_{n}^{k} \boldsymbol{\phi}_{n}=\sum_{n=1}^{N}\left(\sum_{p=0}^{P} \lambda_{n p}^{k} \Psi_{p}(\boldsymbol{\Xi})\right) \boldsymbol{\phi}_{n}
\end{aligned}
$$

where $\left\{a_{p}^{k},\left\{\lambda_{n p}^{k}\right\}_{n=1 \cdots N}\right\}_{p=0 \cdots P}$ are identified according to a procedure proposed in (Dessombz et al. 1999).

\section{EXAMPLE}




\section{2-DOF random system}

Stiffnesses $k_{1}$ and $k_{2}$ of the 2-DOF system shown in Fig. 1 are random variables with

$$
\begin{aligned}
& k_{1}=\bar{k}\left(1+\delta_{K} \xi_{1}\right) \\
& k_{2}=\bar{k}\left(1+\delta_{K} \xi_{2}\right)
\end{aligned}
$$

where random variables $\xi_{1}$ and $\xi_{2}$ are independent; $\bar{k}=15000 \mathrm{~N} / \mathrm{m}, \delta_{K}=5 \%$. The other characteristics are $m=1 \mathrm{~kg}$ and $c=1 \mathrm{~kg} . \mathrm{s}^{-1}$. The two deterministic eigenvalues (i.e. for $\xi_{1}=\xi_{2}=0$ ) are $12.05 \mathrm{~Hz}$ and $31.54 \mathrm{~Hz}$, and the modal damping ratios are $0.25 \%$ and $0.66 \%$.

Mass 1 is excited by force $F_{1}(t)=F_{0} \sin \left(\omega_{e} t\right)$ if $t \in\left[0,2 \pi / \omega_{e}\right]$ and $F_{1}(t)=0$ otherwise; $F_{2}(t)$ is equal to zero; $F_{0}=1 \mathrm{~N} ; \omega_{e}=2.5 \mathrm{rad} / \mathrm{s}$ but two other values were also investigated.

The uncertain stiffness matrix is $\mathbf{K}=\mathbf{K}_{0}+\xi_{1} \mathbf{K}_{1}+\xi_{2} \mathbf{K}_{2}$.

Both random variables $\xi_{1}$ and $\xi_{2}$ follow a normal distribution truncated at \pm 5 standard deviations so that the stiffnesses are positive.

\section{Statistics in the time domain}

The history of the first two statistical moments (mean and standard deviation) were evaluated in several ways. The reference quantities are obtained with a Monte Carlo simulation, where the responses are calculated for each sample of the stiffness matrix; a latin hypercube sampling method with 10000 samples were used to get the samples.

There are two ways to calculate the response: the most usual method consists in integrating motion equation (1); however it is also possible to solve it in the frequency domain by applying a Fourier transform, then dividing the two signals, and finally returning to the time domain with an inverse Fourier transform. The latter method may be much faster than the direct integration, although appropriate signal processing must be applied (Doyle 1997) as well as specific procedures to account for non-zero initial conditions (Siqueira Meirelles and Arruda 2005).

First, the results have been calculated by integrating motion equation (1) with a $\beta-\gamma$-Newmark 
method $(\beta=1 / 4, \gamma=1 / 2$ : constant average acceleration) over the interval $[0,20] \mathrm{s}$ with a time step equal to $0.01 \mathrm{~s}$. The reference results obtained with the Monte Carlo simulation are represented by a blue line in all the following figures. Similarly the PCE (resp. XPA, RM) results are represented by a red (resp. black, magenta) dotted line. A relative error $\operatorname{err}_{S}$ is calculated to compare the reference results, $M C S$, and the results obtained from method $S$ (where $S$ is obtained with either PCE, XPA or $R M)$ :

$$
\operatorname{err}_{S}=\frac{\|S-M C S\|_{2}}{\|M C S\|_{2}}
$$

All the errors are listed in Table 1.

The PCE had an order equal to 15 (which means 136 terms in the expansion). The error is quite high (greater than $18 \%$ ) for the first two statistical moments (see Figs. 2(a) and 3(a)). The XPA results are presented in Figs. 2(b) and 3(b) for $N_{1}=2$ and $D_{1}=2$ : the figures and the errors show that the method is not acceptable. Higher degrees for both the numerator and the denominators were unsuccessfully used. On the contrary the random modes determined with $\mathrm{PC}$ of order equal to 1 were very efficient to estimate the statistics of the response: in Figs. 2(c) and 3(c), it is not possible to distinguish the random mode results from the MCS results.

The PDF of the response at $t=6 \mathrm{~s}$ are shown in Fig. 4 and the quality of the approximation is assessed by calculating the Kullback-Leibler (KL) divergence (Kullback and Leibler 1951; Basseville 2013), $D_{K L}$. It was found that $D_{K L}$ is greater than 20 for both the PCE and the XPA, whereas $D_{K L}=3 \times 10^{-3}$ for the random modes. The results show that the PDF obtained with the random modes are in good agreement with the MCS, whereas the PDFs estimated with the PCE and the XPA are in poor agreement with the MCS. Accordingly, the only efficient method is the random modes method.

It seems that integrating the motion differential equation, Eq. (6), produces a cumulative error from all the previous times on all the coefficients of the PCE: this error spoils the sequence of the coefficients at a given instant. However the extended Padé approximant technique has proved its efficiency for estimating the PDF of the steady-state response (Jacquelin et al. 2017), and hence an investigation of the XPA method based on PC coefficients obtained in the frequency domain is 
proposed.

\section{Statistics in frequency domain}

The Padé approximant technique is therefore applied in frequency domain together with a Monte Carlo simulation (MCS) to calculate a sample of responses in the frequency domain: an inverse Fourier transform transfers all the responses from the frequency domain to the time domain.

In the frequency domain, a PCE of the Fourier transform of the response, $\mathcal{X}$, is carried out

$$
\mathcal{X}(\omega, \Xi)=\sum_{j=0}^{P} \mathcal{Y}_{j}(\omega) \Psi_{j}(\Xi)
$$

and substituted into the Fourier transform of the motion equation, which in turn is projected on each of the PC; this Galerkin projection provides coefficients $\mathcal{Y}_{j}(\omega)$ (Jacquelin et al. 2015). Coefficients $\mathbf{Y}_{j}(t)$ are obtained by applying an inverse Fourier transform to $\mathcal{Y}_{j}(\omega)$

Further, $\left\{\mathcal{Y}_{j}(\omega)\right\}_{\{j=0 \cdots P\}}$ can be used to calculate the XPA of $\mathcal{X}(\omega, \Xi),[M / N]_{\mathcal{X}}(\omega, \Xi)$, in the frequency domain. Therefore the inverse Fourier transform of the XPA is an estimate of $\mathbf{x}(t, \Xi)$.

For completeness, the modal equations were also solved in the frequency domain for all the random modes: an inverse Fourier transform gives the random mode solution in the time domain.

The first two statistical moments were estimated, and the errors with respect to the MCS simulations are listed in Table 2 . The results show that the XPA gives very accurate estimates of the mean and the standard deviation of the response, whereas the accuracy of the PCE and the random modes method is not modified by solving the equations in the frequency domain. Similarly the KL divergence at $t=6 \mathrm{~s}$ is now reduced to $5 \times 10^{-3}$. This is confirmed by the history of the first two statistics and the PDF at $t=6 \mathrm{~s}$, which are plotted in Fig. 5: it is not possible to distinguish the MCS curves and the XPC curves.

In (Jacquelin et al. 2015), it was shown that in case of a harmonic excitation, the PCE model is not efficient for a frequency close to the deterministic eigenfrequencies, tand hence the results for two other frequency are also given in Table $2 . \omega_{e}=75.7 \mathrm{rad} / \mathrm{s}(12 \mathrm{~Hz})$ is close to to the first eigenfrequency and $\omega_{e}=125.7 \mathrm{rad} / \mathrm{s}(20 \mathrm{~Hz})$ is a frequency between the two eigenfrequencies. It can 
be seen that the quality of the results has not deteriorated.

\section{CONCLUSION}

Linear random multiple DOF systems were addressed in this study. It was shown that the random modes were very efficient to estimate the statistics of the transient response. Conversely, the Padé approximant method based on polynomial chaos did not give satisfactory results.

It has been shown in a previous work that XPA is efficient in the frequency domain; hence the $\mathrm{XPA}$ is calculated in the frequency domain and then transformed into the time domain by an inverse Fourier transform. The results were then in excellent agreement with the ones obtained with Monte Carlo simulations. The proposed methods are alternatives to the TDgPC presented in (Gerritsma et al. 2010).

One issue not addressed in this paper is the presence of close modes, which can give problems in the estimation of the PDFs of the random modes. The example had well separated modes and hence the random modes approach worked well, but this is not guaranteed with close modes. Of course the frequency domain approach using the XPA does not require any modal transformation and hence will work equally well for systems with close modes.

\section{REFERENCES}

Baker, G. A. and Graves-Morris, P. (1996). Padé approximants - second edition. Cambridge University press.

Basseville, M. (2013). "Divergence measures for statistical data processing - An annotated bibliography." Signal Processing, 93, 621-633.

Beran, P., Pettit, C., and Millman, D. (2006). "Uncertainty quantification of limit-cycle oscillations." Journal of Computational Physics, 217, 217-247.

Chantrasmi, T., Doostan, A., and Iaccarino, G. (2009). "Padé-Legendre approximants for uncertainty analysis with discontinuous response surfaces." Journal of Computational Physics, 228, 7159-7180.

Dessombz, O., Diniz, A., Thouverez, F., and Jézéquel, L. (1999). "Analysis of stochastic structures: Pertubation method and projection on homogeneous chaos." 7th International Modal Analysis Conference IMAC-SEM, Kissimmee, Floride-USA (February). 
Doyle, J. F. (1997). Wave propagation in structures: spectral analysis using fast discrete Fourier transforms - second edition. Springer-Verlag.

Emmel, L., Kaber, S. M., and Maday, Y. (2003). "Padé-Jacobi filtering for spectral approximations of discontinuous solutions." Numerical Algorithms, 33(1), 251-264.

Gerritsma, M., van der Steen, J.-B., Vos, P., and Karniadakis, G. (2010). "Time-dependent generalized polynomial chaos." Journal of Computational Physics, 229, 8333-8363.

Ghanem, R. G. and Spanos, P. D. (1991). Stochastic Finite Elements: A Spectral Approach. SpringerVerlag, New York, USA.

Guillaume, P., Huard, A., and Robin, V. (2000). "Multivariate Padé approximation." Journal of Computational and Applied Mathematics, 121, 197-219.

Jacquelin, E., Adhikari, S., Sinou, J.-J., and Friswell, M. I. (2015). "The polynomial chaos expansion and the steady-state response of a class of random dynamic systems." Journal of Engineering Mechanics, 141(4), 04014145.

Jacquelin, E., Dessombz, O., Sinou, J.-J., Adhikari, S., and Friswell, M. I. (2017). "Polynomial chaos based eXtended Padé expansion in structural dynamics." International Journal for Numerical Methods in Engineering, 111(12), 1170-1291.

Kullback, S. and Leibler, R. A. (1951). "On information and sufficiency." Annals of Mathematical Statistics, 22(1), 79-86.

Le Maître, O., Mathelin, L., Knio, O., and Hussaini, M. (2010). "Asynchronous time integration for polynomial chaos expansion of uncertain periodic dynamics.” Discret. Contin. Dyn. Sys. - Series A (DCDS-A), 28(1), 199-226.

Le Meitour, J., Lucor, D., and Chassaing, J.-C. (2010). "Prediction of stochastic limit cycle oscillations using an adaptive polynomial chaos method." Journal of Aeroelasticity and Structural Dynamics, 2(1), 3-22.

Matos, A. C. (1996). "Some convergence results for the generalized Padé-type approximants." Numerical Algorithms, 11(1), 255-269.

Siqueira Meirelles, P. and Arruda, J. (2005). "Transient response with arbitrary initial conditions 
$262 \quad$ using the dft." Journal of Sound and Vibration, 287, 525-543.

263 Wiener, N. (1938). "The homogeneous chaos." American Journal of Mathematics, 60(4), 897-936.

${ }_{264}$ Witteveen, J. and Bijl, H. (2006). "Modeling arbitrary uncertainties using gram-schmidt polynomial 265 chaos." Vol. AIAA-2006-896, 44th AIAA Aerospace Sciences Meeting, Reno, Nevada.

${ }_{266}$ Yaghoubi, V., Marelli, S., Sudret, B., and Abrahamsson, T. (2017). "Sparse polynomial chaos ex267 pansions of frequency response functions using stochastic frequency transformation." Probabilistic Engineering Mechanics, 48, 39-58. 


\section{$269 \quad$ List of Tables}

1 Error on some statistics: time domain . . . . . . . . . . . . . . . . . . . . . 14

2 Error on some statistics: frequency domain . . . . . . . . . . . . . . . . . . . 15 
TABLE 1. Error on some statistics: time domain

\begin{tabular}{lcc}
\hline method & mean (\%) & standard deviation (\%) \\
\hline PCE (15) & 18.6 & 19.4 \\
Padé $[1 / 2]$ & 330 & $5 \times 10^{4}$ \\
RM & 0.23 & 0.31 \\
\hline
\end{tabular}


TABLE 2. Error on some statistics: frequency domain

\begin{tabular}{clcc}
\hline$\omega_{e}(\mathrm{rad} / \mathrm{s})$ & method & mean $(\%)$ & std (\%) \\
\hline \multirow{3}{*}{2.5} & PCE $(15)$ & 17.57 & 19.73 \\
& Padé $[1 / 2]$ & $2 \times 10^{-2}$ & $3 \times 10^{-2}$ \\
& RM & 0.26 & 0.23 \\
\hline \multirow{3}{*}{75.7} & PCE $(15)$ & 53.57 & 19.86 \\
& Padé $[1 / 2]$ & $6 \times 10^{-2}$ & $3 \times 10^{-2}$ \\
& RM & 0.80 & 0.21 \\
\hline \multirow{3}{*}{125.7} & PCE $(15)$ & 53.99 & 19.89 \\
& Padé $[1 / 2]$ & $8 \times 10^{-2}$ & $2 \times 10^{-2}$ \\
& RM & 0.81 & 0.20 \\
\hline
\end{tabular}




\section{List of Figures}

1 A two degree-of-freedom system with random stiffnesses . . . . . . . . . . . . . 17

2 Time history of $x_{1}$ mean; MCS (blue solid line) vs. (a): PCE (degree 15, $P=135$ red line); (b): XPA ([1/2], $P=14$ - black line); (c): random modes solution (magenta

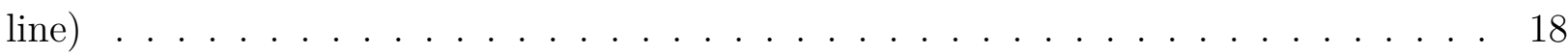

3 Time history of $x_{1}$ standard deviation; MCS (solid blue line) vs. (a): PCE (degree 15, $P=135$ - red line); (b): XPA ([1/2], $P=14$ - black line); (c): random modes solution $($ magenta line $) \ldots \ldots \ldots \ldots$

4 PDF of $x_{1}$ at $t=6 \mathrm{~s}$; MCS (solid blue line) vs. (a): PCE (degree $15, P=135-$ red line); (b): XPA ([1/2], $P=14$ - black line); (c): random modes solution (magenta line) 20

5 statistics of $x_{1}$ obtained through time-frequency transformations; MCS (solid blue line) vs XPA ([1/2], $P=14$ - black line); (a): history of mean; (b): history of standard deviation; (c): PDF of $x_{1}$ at $t=6 \mathrm{~s} \ldots \ldots \ldots \ldots \ldots \ldots$ 


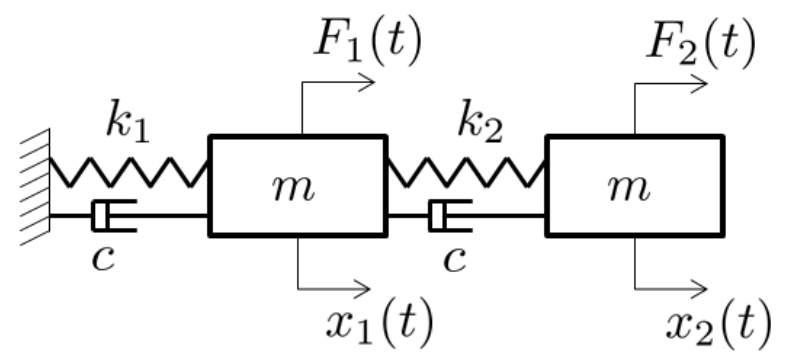

FIG. 1. A two degree-of-freedom system with random stiffnesses 


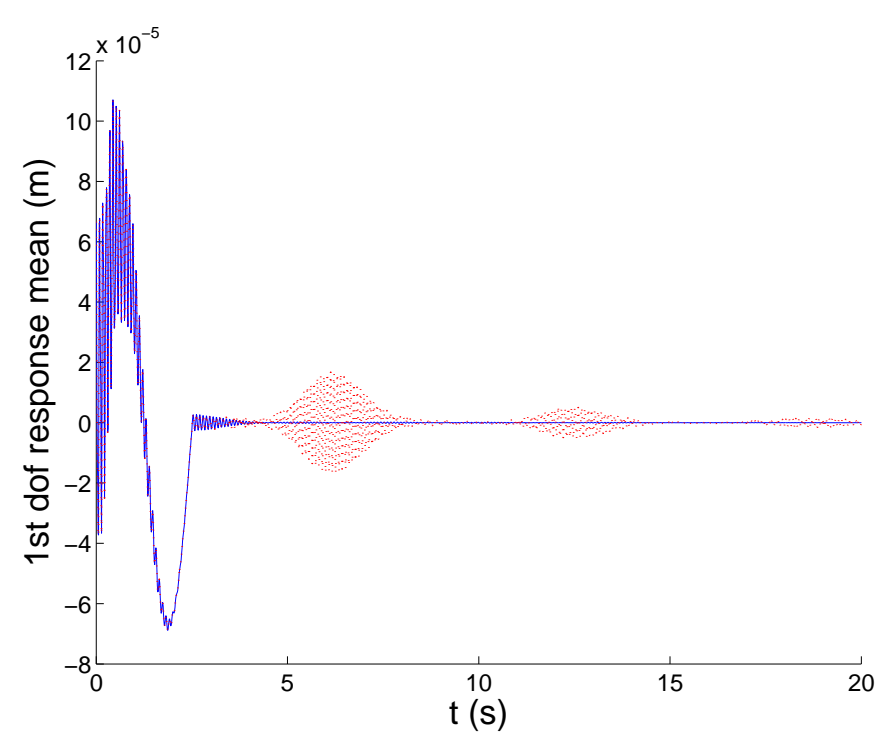

(a)

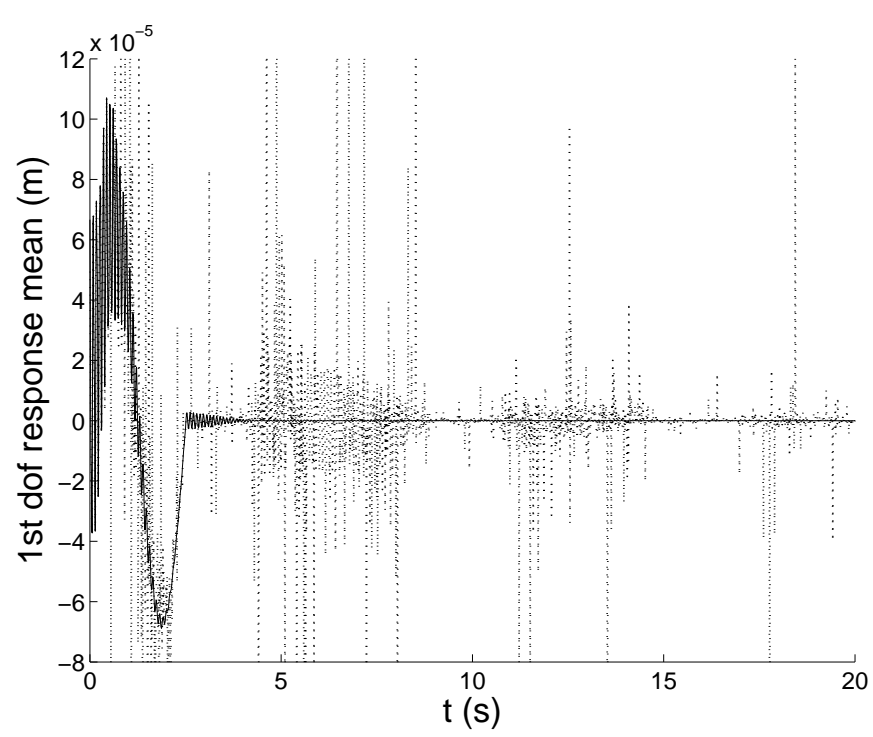

(b)

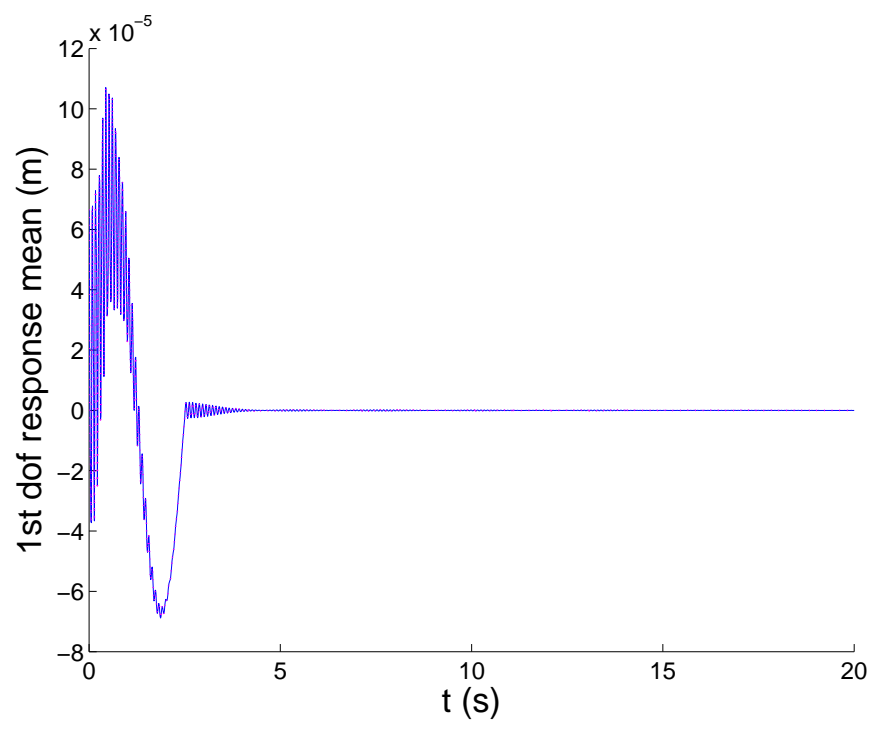

(c)

FIG. 2. Time history of $x_{1}$ mean; MCS (blue solid line) vs. (a): PCE (degree 15, $P=135$ red line); (b): XPA ([1/2], $P=14$ - black line); (c): random modes solution (magenta line) 


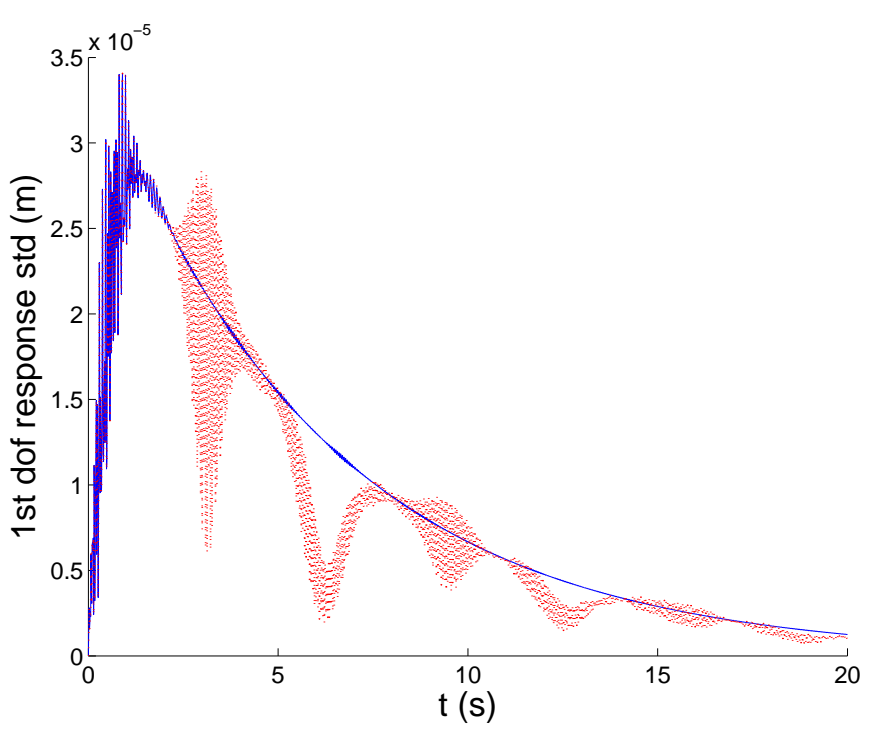

(a)

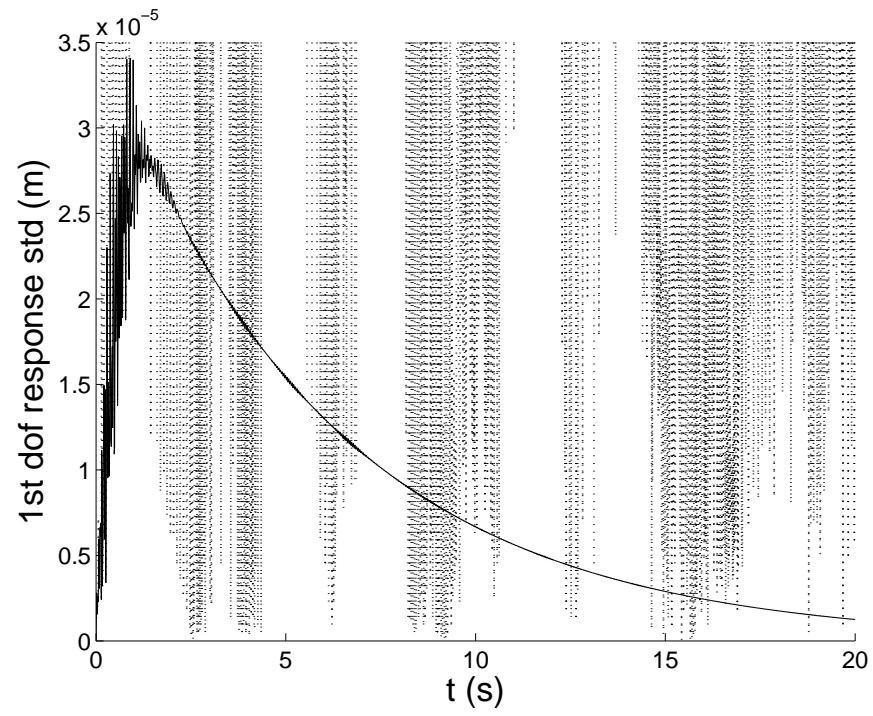

(b)

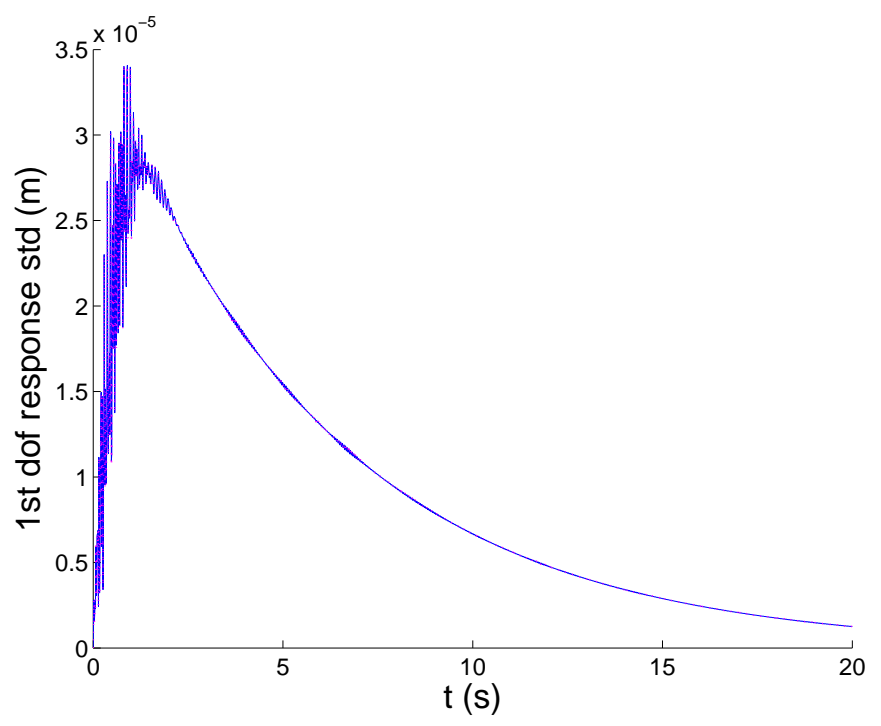

(c)

FIG. 3. Time history of $x_{1}$ standard deviation; MCS (solid blue line) vs. (a): PCE (degree 15, $P=135$ - red line); (b): XPA ([1/2], $P=14$ - black line); (c): random modes solution (magenta line) 


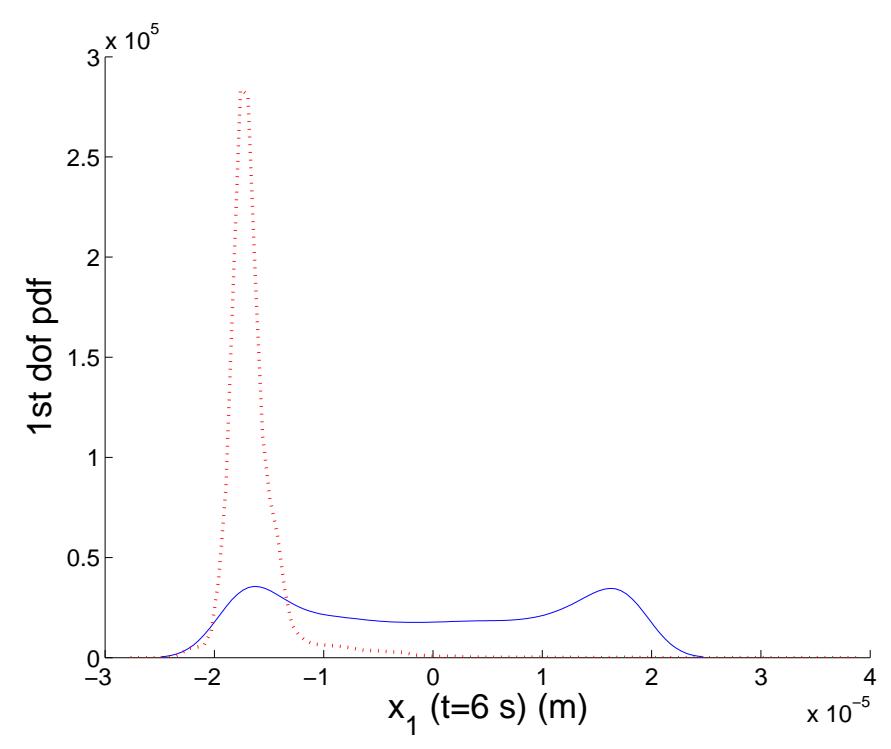

(a)

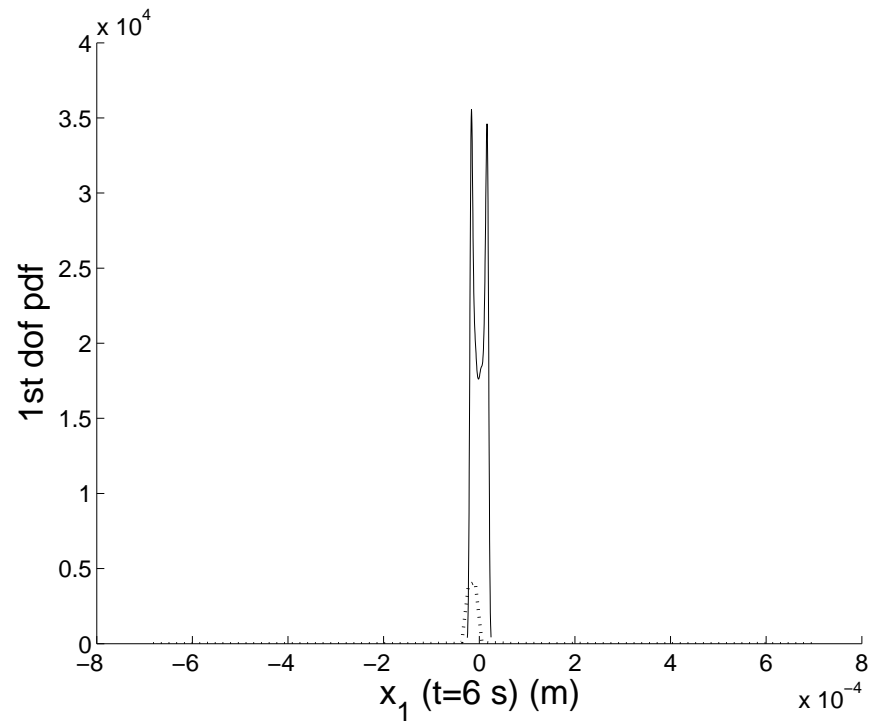

(b)

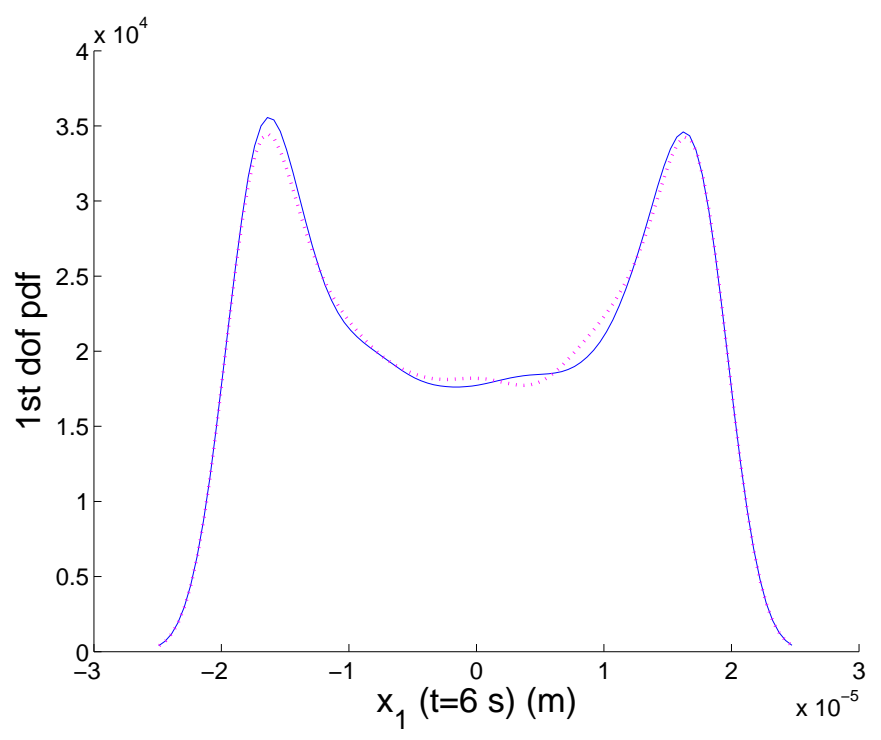

(c)

FIG. 4. PDF of $x_{1}$ at $t=6 \mathrm{~s}$; MCS (solid blue line) vs. (a): PCE (degree 15, $P=135$ - red line); (b): XPA ([1/2], $P=14$ - black line); (c): random modes solution (magenta line) 


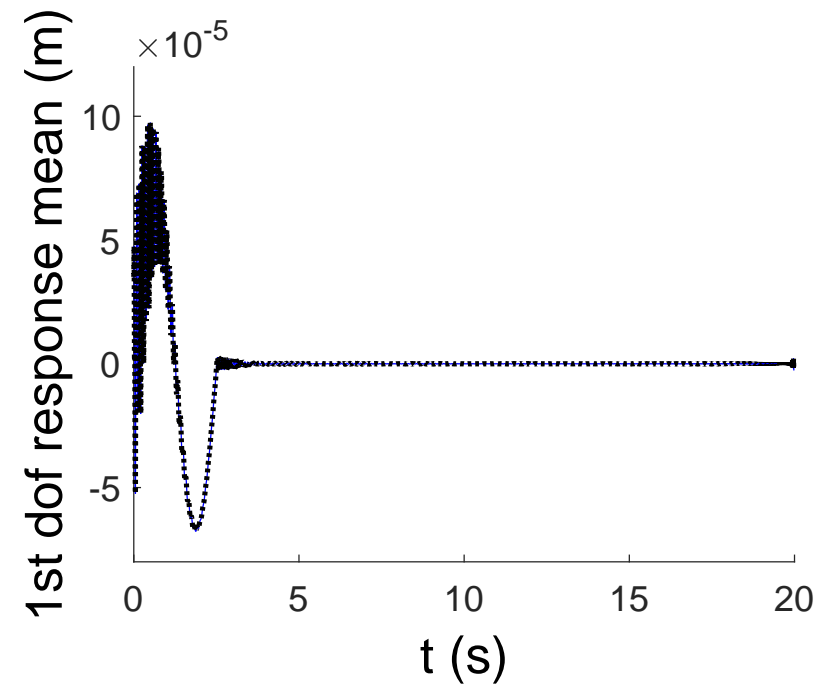

(a)

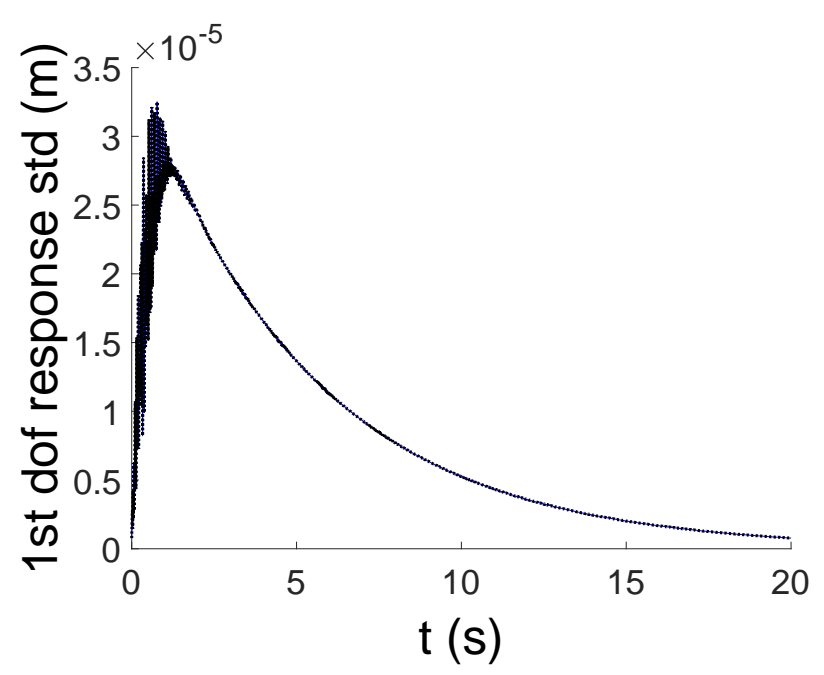

(b)

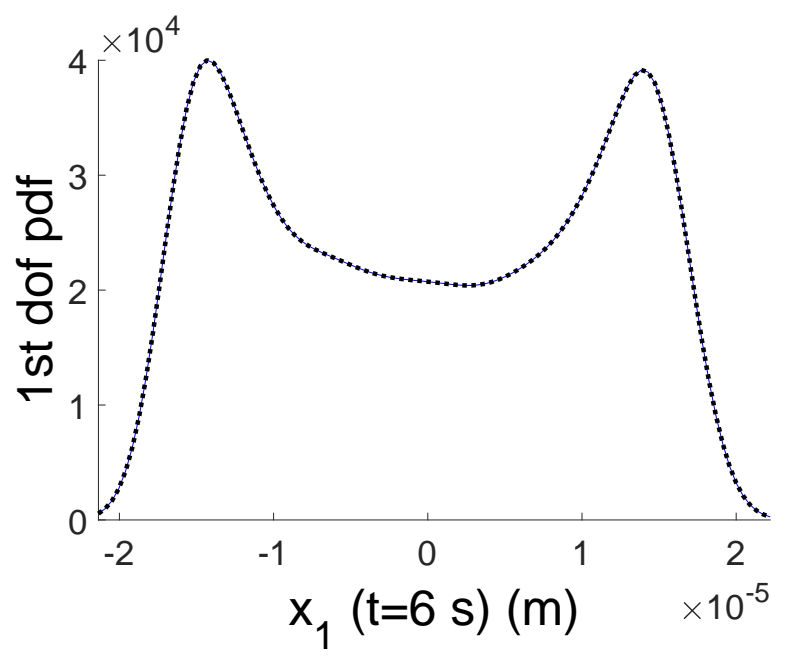

(c)

FIG. 5. statistics of $x_{1}$ obtained through time-frequency transformations; MCS (solid blue line) vs XPA ([1/2], $P=14$ - black line); (a): history of mean; (b): history of standard deviation; (c): PDF of $x_{1}$ at $t=6 \mathbf{s}$ 